Supporting Information for:

\title{
Solid-state NMR Fermi Contact and Dipolar Shifts in Organometallic Complexes and Metalloporphyrins
}

\author{
Yong Zhang, Haihong Sun, and Eric Oldfield* \\ Departments of Chemistry and Biophysics, University of Illinois at Urbana-Champaign, 600 South Mathews
}

Avenue, Urbana, IL 61801, USA

\section{Full Citation of Gaussian 03 in Ref.11:}

Gaussian 03, Revision B.03; Frisch, M. J.; Trucks, G. W.; Schlegel, H. B.; Scuseria, G. E.; Robb, M. A.; Cheeseman, J. R.; Montgomery, Jr., J. A.; Vreven, T.; Kudin, K. N.; Burant, J. C.; Millam, J. M.; Iyengar, S. S.; Tomasi, J.; Barone, V.; Mennucci, B.; Cossi, M.; Scalmani, G.; Rega, N.; Petersson, G. A.; Nakatsuji, H.; Hada, M.; Ehara, M.; Toyota, K.; Fukuda, R.; Hasegawa, J.; Ishida, M.; Nakajima, T.; Honda, Y.; Kitao, O.; Nakai, H.; Klene, M.; Li, X.; Knox, J. E.; Hratchian, H. P.; Cross, J. B.; Adamo, C.; Jaramillo, J.; Gomperts, R.; Stratmann, R. E.;Yazyev, O.; Austin, A. J.; Cammi, R.; Pomelli, C.; Ochterski, J. W.; Ayala, P. Y.; Morokuma, K.; Voth, G. A.; Salvador, P.; Dannenberg, J. J.; Zakrzewski, V. G.; Dapprich, S.; Daniels, A. D.; Strain, M. C.; Farkas, O.; Malick, D. K.; Rabuck, A. D.; Raghavachari, K.; Foresman, J. B.; Ortiz, J. V.; Cui, Q.; Baboul, A. G.; Clifford, S.; Cioslowski, J.; Stefanov, B. B.; Liu, G.; Liashenko, A.; Piskorz, P.; Komaromi, I.; Martin, R. L.; Fox, D. J.; Keith, T.; Al-Laham, M. A.; Peng, C. Y.; Nanayakkara, A.; Challacombe, M.; Gill, P. M. W.; Johnson, B.; Chen, W.; Wong, M. W.; Gonzalez, C.; Pople, J. A.; Gaussian, Inc., Pittsburgh PA, 2003.

\section{Computational Details:}

The details of computational aspects are basically the same as we used before (see Refs.9 and 11-12), except that for 1, a cluster approach was used due to its polymeric structure nature as revealed by the x-ray crystallography. The basis set schemes used for different cluster models for 1 were described in Ref.15. As before, SGI O300 workstations were employed in our calculations using up to 8 CPUs. The most important aspect of these calculations lies in the design of an appropriate structural model of the $\mathrm{x}$-ray structure to mimic the real metal complex system. The 5-coordinate $\mathrm{Cu}-1$ model contains one $\mathrm{Cu}(\mathrm{DL}-\mathrm{ala})_{2}$ unit with one axially coordinated water, as expected in previous studies. The other axially coordinated water was incorporated in the 6-coordinate $\mathrm{Cu}-1 \mathrm{model}$. $\mathrm{So}$, in this 6-coordinate $\mathrm{Cu}-1$ model, the copper coordination is complete. To consider copper-copper interactions along the polymer chain, a 6-coordinate $\mathrm{Cu}-3$ model was used. Two $\mathrm{Cu}(\mathrm{DL}-\mathrm{ala})_{2}$ units coordinated to the two axial water molecules in the central unit $\left(\mathrm{H}_{2} \mathrm{O}-\mathrm{Cu}-\mathrm{H}_{2} \mathrm{O}\right)$ of the 6-coordinate $\mathrm{Cu}-1$ model were included in the calculation, forming a $\mathrm{Cu}-\mathrm{H}_{2} \mathrm{O}-\mathrm{Cu}-\mathrm{H}_{2} \mathrm{O}-\mathrm{Cu}$ structure. However, as shown in Figure $2 \mathrm{~A}$, the shift deviation is still large, especially for the methyl carbon, which suggests that the inter-chain interaction is not negligible. In fact, the $\mathrm{x}$-ray structure shows that the central unit $\left(\mathrm{H}_{2} \mathrm{O}-\mathrm{Cu}-\mathrm{H}_{2} \mathrm{O}\right)$ has 14 hydrogen bonds between eight neighboring $\mathrm{Cu}(\mathrm{DL}-\mathrm{ala})_{2}$ and two water molecules. Therefore, this central unit, together with the hydrogen bonded neighboring $\mathrm{Cu}(\mathrm{DL}-\mathrm{ala})_{2}$ and water molecules, were considered in the $\mathrm{Cu}-9$ cluster. In the following Table, we present the results of spin densities of both carbon and hydrogen atoms of interest, calculated in the different models. It is clear that the ${ }^{13} \mathrm{C}$ spin densities (and hyperfine shifts) suffer most from the incomplete consideration of metal-metal interactions, which is graphically demonstrated in Figure 2A.

Table S1. Computed Spin Densities of 1 in Different Models (unit: au)

\begin{tabular}{llcccc}
\hline Nucleus & ${ }^{2}$ & $\begin{array}{c}\text { 5-coordinate } \\
\text { Cu-1 }\end{array}$ & $\begin{array}{c}\text { 6-coordinate } \\
\text { Cu-1 }\end{array}$ & $\begin{array}{c}\text { 6-coordinate } \\
\text { Cu-3 }\end{array}$ & $\begin{array}{c}\text { 6-coordinate } \\
\text { Cu-9 }\end{array}$ \\
\hline & & -0.00349 & -0.00329 & -0.00333 & -0.00323 \\
$\mathbf{1}$ & ${ }^{13} \mathbf{C H}$ & -0.00511 & -0.0483 & -0.00457 & -0.00400 \\
$\mathbf{1}$ & ${ }^{13} \mathbf{C O}_{2}^{-}$ & -0.000073 & 0.00096 & 0.00138 \\
$\mathbf{1}$ & ${ }^{13} \mathbf{C H}_{3}$ & 0.00069 & -0.00134 & -0.00138 & -0.00142 \\
$\mathbf{1}$ & $\mathrm{N}^{1} \mathbf{H}_{2}$ & -0.00137 & 0.00000 & 0.00000 & 0.00000 \\
$\mathbf{1}$ & $\mathrm{C}^{1} \mathbf{H}^{1}$ & -0.00001 & 0.00030 & 0.00030 & 0.00026 \\
\hline
\end{tabular}

\footnotetext{
${ }^{a}$ The nucleus of interest is in bold.
} 
The largest structure (Cu-9) investigated has 1628 basis functions and the DFT calculation took approximately 130 CPU hours. For the convenience of the reader, the Gaussian input file of the $\mathrm{Cu}-9$ cluster calculation is provided below:

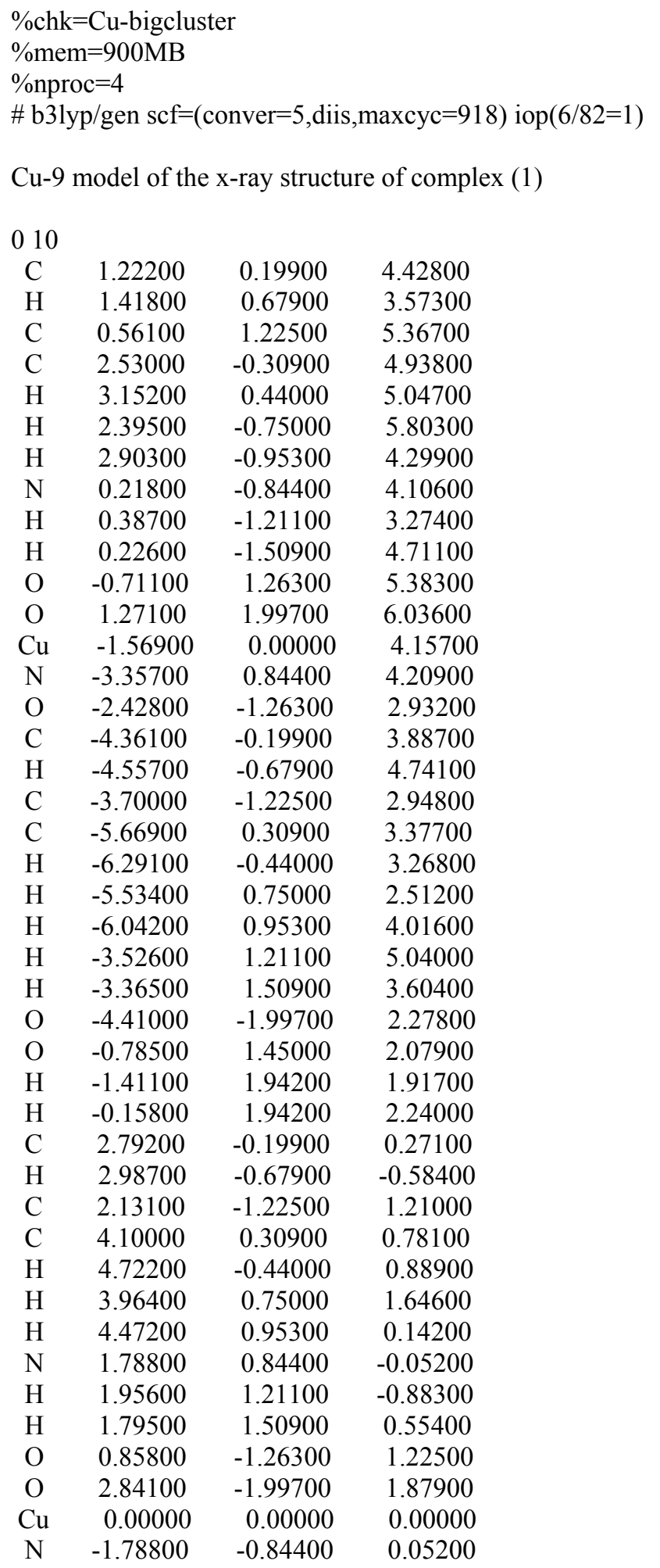




\begin{tabular}{|c|c|c|c|}
\hline $\mathrm{O}$ & -0.85800 & 1.26300 & -1.22500 \\
\hline $\mathrm{C}$ & -2.79200 & 0.19900 & -0.27100 \\
\hline $\mathrm{H}$ & -2.98700 & 0.67900 & 0.58400 \\
\hline C & -2.13100 & 1.22500 & -1.21000 \\
\hline $\mathrm{C}$ & -4.10000 & -0.30900 & -0.78100 \\
\hline $\mathrm{H}$ & -4.72200 & 0.44000 & -0.88900 \\
\hline $\mathrm{H}$ & -3.96400 & -0.75000 & -1.64600 \\
\hline $\mathrm{H}$ & -4.47200 & -0.95300 & -0.14200 \\
\hline $\mathrm{H}$ & -1.95600 & -1.21100 & 0.88300 \\
\hline $\mathrm{H}$ & -1.79500 & -1.50900 & -0.55400 \\
\hline $\mathrm{O}$ & -2.84100 & 1.99700 & -1.87900 \\
\hline $\mathrm{C}$ & 11.71400 & -0.19900 & 8.58500 \\
\hline $\mathrm{H}$ & 11.90900 & -0.67900 & 7.73000 \\
\hline $\mathrm{C}$ & 11.05300 & -1.22500 & 9.52400 \\
\hline $\mathrm{C}$ & 13.02200 & 0.30900 & 9.09500 \\
\hline $\mathrm{H}$ & 13.64400 & -0.44000 & 9.20400 \\
\hline $\mathrm{H}$ & 12.88600 & 0.75000 & 9.96000 \\
\hline $\mathrm{H}$ & 13.39400 & 0.95300 & 8.45600 \\
\hline $\mathrm{N}$ & 10.71000 & 0.84400 & 8.26300 \\
\hline $\mathrm{H}$ & 10.87900 & 1.21100 & 7.43200 \\
\hline $\mathrm{H}$ & 10.71700 & 1.50900 & 8.86800 \\
\hline $\mathrm{O}$ & 9.78000 & -1.26300 & 9.54000 \\
\hline $\mathrm{O}$ & 11.76300 & -1.99700 & 10.19300 \\
\hline $\mathrm{Cu}$ & 8.92200 & 0.00000 & 8.31500 \\
\hline $\mathrm{N}$ & 7.13400 & -0.84400 & 8.36600 \\
\hline $\mathrm{O}$ & 8.06400 & 1.26300 & 7.08900 \\
\hline $\mathrm{C}$ & 6.13000 & 0.19900 & 8.04400 \\
\hline $\mathrm{H}$ & 5.93500 & 0.67900 & 8.89900 \\
\hline $\mathrm{C}$ & 6.79100 & 1.22500 & 7.10500 \\
\hline $\mathrm{C}$ & 4.82200 & -0.30900 & 7.53400 \\
\hline $\mathrm{H}$ & 4.20000 & 0.44000 & 7.42500 \\
\hline $\mathrm{H}$ & 4.95800 & -0.75000 & 6.66900 \\
\hline $\mathrm{H}$ & 4.45000 & -0.95300 & 8.17300 \\
\hline $\mathrm{H}$ & 6.96600 & -1.21100 & 9.19700 \\
\hline $\mathrm{H}$ & 7.12700 & -1.50900 & 7.76100 \\
\hline $\mathrm{O}$ & 6.08100 & 1.99700 & 6.43600 \\
\hline $\mathrm{C}$ & 2.79200 & 9.36600 & 0.27100 \\
\hline $\mathrm{H}$ & 2.98700 & 8.88500 & -0.58400 \\
\hline $\mathrm{C}$ & 2.13100 & 8.33900 & 1.21000 \\
\hline $\mathrm{C}$ & 4.10000 & 9.87400 & 0.78100 \\
\hline $\mathrm{H}$ & 4.72200 & 9.12400 & 0.88900 \\
\hline $\mathrm{H}$ & 3.96400 & 10.31400 & 1.64600 \\
\hline $\mathrm{H}$ & 4.47200 & 10.51700 & 0.14200 \\
\hline $\mathrm{N}$ & 1.78800 & 10.40800 & -0.05200 \\
\hline $\mathrm{H}$ & 1.95600 & 10.77500 & -0.88300 \\
\hline $\mathrm{H}$ & 1.79500 & 11.07300 & 0.55400 \\
\hline $\mathrm{O}$ & 0.85800 & 8.30100 & 1.22500 \\
\hline $\mathrm{O}$ & 2.84100 & 7.56700 & 1.87900 \\
\hline $\mathrm{Cu}$ & 0.00000 & 9.56400 & 0.00000 \\
\hline $\mathrm{N}$ & -1.78800 & 8.72000 & 0.05200 \\
\hline $\mathrm{O}$ & -0.85800 & 10.82700 & -1.22500 \\
\hline $\mathrm{C}$ & -2.79200 & 9.76300 & -0.27100 \\
\hline $\mathrm{H}$ & -2.98700 & 10.24400 & 0.58400 \\
\hline $\mathrm{C}$ & -2.13100 & 10.78900 & -1.21000 \\
\hline $\mathrm{C}$ & -4.10000 & 9.25500 & -0.78100 \\
\hline $\mathrm{H}$ & -4.72200 & 10.00400 & -0.88900 \\
\hline
\end{tabular}




\begin{tabular}{|c|c|c|c|}
\hline $\mathrm{H}$ & -3.96400 & 8.81500 & -1.64600 \\
\hline $\mathrm{H}$ & -4.47200 & 8.61200 & -0.14200 \\
\hline $\mathrm{H}$ & -1.95600 & 8.35300 & 0.88300 \\
\hline $\mathrm{H}$ & -1.79500 & 8.05500 & -0.55400 \\
\hline $\mathrm{O}$ & -2.84100 & 11.56100 & -1.87900 \\
\hline $\mathrm{C}$ & 8.82200 & 4.58400 & 0.27100 \\
\hline $\mathrm{H}$ & 9.01800 & 4.10300 & -0.58400 \\
\hline $\mathrm{C}$ & 8.16100 & 3.55700 & 1.21000 \\
\hline $\mathrm{C}$ & 10.13000 & 5.09200 & 0.78100 \\
\hline $\mathrm{H}$ & 10.75200 & 4.34200 & 0.88900 \\
\hline $\mathrm{H}$ & 9.99500 & 5.53200 & 1.64600 \\
\hline $\mathrm{H}$ & 10.50300 & 5.73500 & 0.14200 \\
\hline $\mathrm{N}$ & 7.81800 & 5.62600 & -0.05200 \\
\hline $\mathrm{H}$ & 7.98700 & 5.99300 & -0.88300 \\
\hline $\mathrm{H}$ & 7.82600 & 6.29100 & 0.55400 \\
\hline $\mathrm{O}$ & 6.88900 & 3.51900 & 1.22500 \\
\hline $\mathrm{O}$ & 8.87100 & 2.78500 & 1.87900 \\
\hline $\mathrm{Cu}$ & 6.03100 & 4.78200 & 0.00000 \\
\hline $\mathrm{N}$ & 4.24300 & 3.93800 & 0.05200 \\
\hline $\mathrm{O}$ & 5.17200 & 6.04500 & -1.22500 \\
\hline $\mathrm{C}$ & 3.23900 & 4.98100 & -0.27100 \\
\hline $\mathrm{H}$ & 3.04300 & 5.46100 & 0.58400 \\
\hline $\mathrm{C}$ & 3.90000 & 6.00700 & -1.21000 \\
\hline $\mathrm{C}$ & 1.93100 & 4.47300 & -0.78100 \\
\hline $\mathrm{H}$ & 1.30900 & 5.22200 & -0.88900 \\
\hline $\mathrm{H}$ & 2.06600 & 4.03300 & -1.64600 \\
\hline $\mathrm{H}$ & 1.55800 & 3.83000 & -0.14200 \\
\hline $\mathrm{H}$ & 4.07400 & 3.57100 & 0.88300 \\
\hline $\mathrm{H}$ & 4.23500 & 3.27300 & -0.55400 \\
\hline $\mathrm{O}$ & 3.19000 & 6.77900 & -1.87900 \\
\hline $\mathrm{O}$ & 3.67600 & 3.33200 & 6.23600 \\
\hline $\mathrm{H}$ & 4.30300 & 2.84000 & 6.39700 \\
\hline $\mathrm{H}$ & 3.05000 & 2.84000 & 6.07400 \\
\hline $\mathrm{C}$ & 1.66900 & 4.58400 & 3.88700 \\
\hline $\mathrm{H}$ & 1.47400 & 4.10300 & 4.74100 \\
\hline $\mathrm{C}$ & 2.33000 & 3.55700 & 2.94800 \\
\hline $\mathrm{C}$ & 0.36100 & 5.09200 & 3.37700 \\
\hline $\mathrm{H}$ & -0.26100 & 4.34200 & 3.26800 \\
\hline $\mathrm{H}$ & 0.49700 & 5.53200 & 2.51200 \\
\hline $\mathrm{H}$ & -0.01100 & 5.73500 & 4.01600 \\
\hline $\mathrm{N}$ & 2.67300 & 5.62600 & 4.20900 \\
\hline $\mathrm{H}$ & 2.50500 & 5.99300 & 5.04000 \\
\hline $\mathrm{H}$ & 2.66600 & 6.29100 & 3.60400 \\
\hline $\mathrm{O}$ & 3.60300 & 3.51900 & 2.93200 \\
\hline $\mathrm{O}$ & 1.62000 & 2.78500 & 2.27800 \\
\hline $\mathrm{Cu}$ & 4.46100 & 4.78200 & 4.15700 \\
\hline $\mathrm{N}$ & 6.24900 & 3.93800 & 4.10600 \\
\hline $\mathrm{O}$ & 5.31900 & 6.04500 & 5.38300 \\
\hline $\mathrm{C}$ & 7.25300 & 4.98100 & 4.42800 \\
\hline $\mathrm{H}$ & 7.44800 & 5.46100 & 3.57300 \\
\hline $\mathrm{C}$ & 6.59200 & 6.00700 & 5.36700 \\
\hline $\mathrm{C}$ & 8.56100 & 4.47300 & 4.93800 \\
\hline $\mathrm{H}$ & 9.18300 & 5.22200 & 5.04700 \\
\hline $\mathrm{H}$ & 8.42500 & 4.03300 & 5.80300 \\
\hline $\mathrm{H}$ & 8.93300 & 3.83000 & 4.29900 \\
\hline $\mathrm{H}$ & 6.41800 & 3.57100 & 3.27400 \\
\hline
\end{tabular}




\begin{tabular}{|c|c|c|c|}
\hline $\mathrm{H}$ & 6.25600 & 3.27300 & 4.71100 \\
\hline $\mathrm{O}$ & 7.30200 & 6.77900 & 6.03600 \\
\hline $\mathrm{O}$ & 5.24600 & 6.23300 & 2.07900 \\
\hline $\mathrm{H}$ & 4.61900 & 6.72500 & 1.91700 \\
\hline $\mathrm{H}$ & 5.87200 & 6.72500 & 2.24000 \\
\hline $\mathrm{C}$ & 5.68300 & 4.58400 & 8.58500 \\
\hline $\mathrm{H}$ & 5.87900 & 4.10300 & 7.73000 \\
\hline $\mathrm{C}$ & 5.02200 & 3.55700 & 9.52400 \\
\hline $\mathrm{C}$ & 6.99100 & 5.09200 & 9.09500 \\
\hline $\mathrm{H}$ & 7.61300 & 4.34200 & 9.20400 \\
\hline $\mathrm{H}$ & 6.85600 & 5.53200 & 9.96000 \\
\hline $\mathrm{H}$ & 7.36400 & 5.73500 & 8.45600 \\
\hline $\mathrm{N}$ & 4.67900 & 5.62600 & 8.26300 \\
\hline $\mathrm{H}$ & 4.84800 & 5.99300 & 7.43200 \\
\hline $\mathrm{H}$ & 4.68700 & 6.29100 & 8.86800 \\
\hline $\mathrm{O}$ & 3.75000 & 3.51900 & 9.54000 \\
\hline $\mathrm{O}$ & 5.73200 & 2.78500 & 10.19300 \\
\hline $\mathrm{Cu}$ & 2.89200 & 4.78200 & 8.31500 \\
\hline $\mathrm{N}$ & 1.10400 & 3.93800 & 8.36600 \\
\hline $\mathrm{O}$ & 2.03300 & 6.04500 & 7.08900 \\
\hline $\mathrm{C}$ & 0.10000 & 4.98100 & 8.04400 \\
\hline $\mathrm{H}$ & -0.09600 & 5.46100 & 8.89900 \\
\hline $\mathrm{C}$ & 0.76100 & 6.00700 & 7.10500 \\
\hline $\mathrm{C}$ & -1.20800 & 4.47300 & 7.53400 \\
\hline $\mathrm{H}$ & -1.83000 & 5.22200 & 7.42500 \\
\hline $\mathrm{H}$ & -1.07300 & 4.03300 & 6.66900 \\
\hline $\mathrm{H}$ & -1.58100 & 3.83000 & 8.17300 \\
\hline $\mathrm{H}$ & 0.93500 & 3.57100 & 9.19700 \\
\hline $\mathrm{H}$ & 1.09600 & 3.27300 & 7.76100 \\
\hline $\mathrm{O}$ & 0.05100 & 6.77900 & 6.43600 \\
\hline $\mathrm{O}$ & 9.70700 & 8.11400 & 6.23600 \\
\hline $\mathrm{H}$ & 10.33300 & 7.62200 & 6.39700 \\
\hline $\mathrm{H}$ & 9.08100 & 7.62200 & 6.07400 \\
\hline $\mathrm{C}$ & 7.70000 & 9.36600 & 3.88700 \\
\hline $\mathrm{H}$ & 7.50400 & 8.88500 & 4.74100 \\
\hline $\mathrm{C}$ & 8.36100 & 8.33900 & 2.94800 \\
\hline $\mathrm{C}$ & 6.39200 & 9.87400 & 3.37700 \\
\hline $\mathrm{H}$ & 5.77000 & 9.12400 & 3.26800 \\
\hline $\mathrm{H}$ & 6.52700 & 10.31400 & 2.51200 \\
\hline $\mathrm{H}$ & 6.01900 & 10.51700 & 4.01600 \\
\hline $\mathrm{N}$ & 8.70400 & 10.40800 & 4.20900 \\
\hline $\mathrm{H}$ & 8.53500 & 10.77500 & 5.04000 \\
\hline $\mathrm{H}$ & 8.69600 & 11.07300 & 3.60400 \\
\hline $\mathrm{O}$ & 9.63300 & 8.30100 & 2.93200 \\
\hline $\mathrm{O}$ & 7.65100 & 7.56700 & 2.27800 \\
\hline $\mathrm{Cu}$ & 10.49200 & 9.56400 & 4.15700 \\
\hline $\mathrm{N}$ & 12.27900 & 8.72000 & 4.10600 \\
\hline $\mathrm{O}$ & 11.35000 & 10.82700 & 5.38300 \\
\hline $\mathrm{C}$ & 13.28300 & 9.76300 & 4.42800 \\
\hline $\mathrm{H}$ & 13.47900 & 10.24400 & 3.57300 \\
\hline $\mathrm{C}$ & 12.62200 & 10.78900 & 5.36700 \\
\hline $\mathrm{C}$ & 14.59100 & 9.25500 & 4.93800 \\
\hline $\mathrm{H}$ & 15.21300 & 10.00400 & 5.04700 \\
\hline $\mathrm{H}$ & 14.45600 & 8.81500 & 5.80300 \\
\hline $\mathrm{H}$ & 14.96400 & 8.61200 & 4.29900 \\
\hline $\mathrm{H}$ & 12.44800 & 8.35300 & 3.27400 \\
\hline
\end{tabular}




$\begin{array}{cccc}\mathrm{H} & 12.28700 & 8.05500 & 4.71100 \\ \mathrm{O} & 13.33200 & 11.56100 & 6.03600 \\ \mathrm{C} & 11.71400 & 9.36600 & 8.58500 \\ \mathrm{H} & 11.90900 & 8.88500 & 7.73000 \\ \mathrm{C} & 11.05300 & 8.33900 & 9.52400 \\ \mathrm{C} & 13.02200 & 9.87400 & 9.09500 \\ \mathrm{H} & 13.64400 & 9.12400 & 9.20400 \\ \mathrm{H} & 12.88600 & 10.31400 & 9.96000 \\ \mathrm{H} & 13.39400 & 10.51700 & 8.45600 \\ \mathrm{~N} & 10.71000 & 10.40800 & 8.26300 \\ \mathrm{H} & 10.87900 & 10.77500 & 7.43200 \\ \mathrm{H} & 10.71700 & 11.07300 & 8.86800 \\ \mathrm{O} & 9.78000 & 8.30100 & 9.54000 \\ \mathrm{O} & 11.76300 & 7.56700 & 10.19300 \\ \mathrm{Cu} & 8.92200 & 9.56400 & 8.31500 \\ \mathrm{~N} & 7.13400 & 8.72000 & 8.36600 \\ \mathrm{O} & 8.06400 & 10.82700 & 7.08900 \\ \mathrm{C} & 6.13000 & 9.76300 & 8.04400 \\ \mathrm{H} & 5.93500 & 10.24400 & 8.89900 \\ \mathrm{C} & 6.79100 & 10.78900 & 7.10500 \\ \mathrm{C} & 4.82200 & 9.25500 & 7.53400 \\ \mathrm{H} & 4.20000 & 10.00400 & 7.42500 \\ \mathrm{H} & 4.95800 & 8.81500 & 6.66900 \\ \mathrm{H} & 4.45000 & 8.61200 & 8.17300 \\ \mathrm{H} & 6.96600 & 8.35300 & 9.19700 \\ \mathrm{H} & 7.12700 & 8.05500 & 7.76100 \\ \mathrm{O} & 6.08100 & 11.56100 & 6.43600\end{array}$

1291321341351391421431451461471491501561570

6-311G*

$* * * *$

1301311331361371381401411481511521531541551581590

$6-31 g^{*}$

$* * * *$

1440

S $6 \quad 1.00$

$337200.000 \quad 0.280000000 \mathrm{E}-03$

$50072.9000 \quad 0.217000000 \mathrm{E}-02$

$11373.4000 \quad 0.112200000 \mathrm{E}-01$

$3239.82000 \quad 0.446210000 \mathrm{E}-01$

$1071.97000 \quad 0.138360000$

$395.099000 \quad 0.312550000$

S 21.00

$158.399000 \quad 0.410600000$

$\begin{array}{ll}67.3591000 & 0.214530000\end{array}$

S 11.00

$22.2983000 \quad 1.00000000$

S 11.00

$9.39357000 \quad 1.00000000$

S 11.00

$2.57848000 \quad 1.00000000$

S 11.00

$0.964080000 \quad 1.00000000$

S 11.00

$0.113303000 \quad 1.00000000$

S 111.00 


\begin{tabular}{|c|c|c|}
\hline & $0.407910000 \mathrm{E}-01$ & 1.00000000 \\
\hline \multirow[t]{4}{*}{$\mathrm{P}$} & 31.00 & \\
\hline & 2245.29000 & $0.226000000 \mathrm{E}-02$ \\
\hline & 532.106000 & $0.184100000 \mathrm{E}-01$ \\
\hline & 172.195000 & $0.864600000 \mathrm{E}-01$ \\
\hline \multirow[t]{4}{*}{$\mathrm{P}$} & 31.00 & \\
\hline & 65.3239000 & 0.254500000 \\
\hline & 27.0551000 & 0.434190000 \\
\hline & 11.7435000 & 0.333900000 \\
\hline \multirow[t]{2}{*}{$\mathrm{P}$} & 11.00 & \\
\hline & 4.69382000 & 1.00000000 \\
\hline \multirow[t]{3}{*}{$\mathrm{P}$} & 21.00 & \\
\hline & 1.90667000 & 0.569360000 \\
\hline & 0.711445000 & 0.247020000 \\
\hline \multirow[t]{2}{*}{$\mathrm{P}$} & 11.00 & \\
\hline & 0.232598000 & 1.00000000 \\
\hline \multirow[t]{2}{*}{$\mathrm{P}$} & 11.00 & \\
\hline & $0.692990000 \mathrm{E}-01$ & 1.00000000 \\
\hline \multirow[t]{4}{*}{$\mathrm{D}$} & 31.00 & \\
\hline & 48.5439000 & $0.309700000 \mathrm{E}-01$ \\
\hline & 13.5490000 & 0.161960000 \\
\hline & 4.51628000 & 0.378120000 \\
\hline \multirow[t]{2}{*}{$\mathrm{D}$} & 11.00 & \\
\hline & 1.47329000 & 1.00000000 \\
\hline \multirow[t]{2}{*}{$\mathrm{D}$} & 11.00 & \\
\hline & 0.414875000 & 1.00000000 \\
\hline & 11.00 & \\
\hline & $\underset{\leqslant *}{0.149100000}$ & 1.00000000 \\
\hline
\end{tabular}

12345678910111213141516171819200

$3-21 G^{*}$

****

21222324252627282930313233343536373839400

3-21G*

****

41424344454647484950515253545556575859600

$3-21 \mathrm{G}^{*}$

****

61626364656667686970717273747576777879800

$3-21 \mathrm{G}^{*}$

****

818283848586878889909192939495969798991000

$3-21 G^{*}$

$* * * *$

1011021031041051061071081091101111121131141151161171181191200

$3-21 \mathrm{G}^{*}$

$* * * *$

1211221231241251261271280

$3-21 G^{*}$

$* * * *$

1600

3-21G*

$* * * *$

1611621631641651661671681691701711721731741751761771781791800 $3-21 G^{*}$

$* * * *$ 
1811821831841851861871881891901911921931941951961971981992000 $3-21 G^{*}$

$* * * *$

2012022032042052062072082092102112122132142152162172182192200 $3-21 \mathrm{G}^{*}$

2212222232242252262272282292302312322332342352362370

$3-21 G^{*}$

$* * * *$ 
Table S2. Crystal data and structure refinement for p05yas.

Identification code

Empirical formula

Formula weight

Temperature

Wavelength

Crystal system

Space group

Unit cell dimensions

Volume

Z

Density (calculated)

Absorption coefficient

$\mathrm{F}(000)$

Crystal size

Theta range for data collection

Index ranges

Reflections collected

Independent reflections

Completeness to theta $=25.47^{\circ}$

Absorption correction

Max. and min. transmission

Refinement method

Data / restraints / parameters

Goodness-of-fit on $\mathrm{F}^{2}$

Final $\mathrm{R}$ indices [I $>2 \operatorname{sigma}(\mathrm{I})]$

$\mathrm{R}$ indices (all data)

Largest diff. peak and hole p05yas

C6 H14 Cu N2 O5

257.73

193(2) K

$0.71073 \AA$

Monoclinic

C $2 / \mathrm{c}$

$\mathrm{a}=12.061(5) \AA$

$\alpha=90^{\circ}$.

$\mathrm{b}=9.564(4) \AA$

$\beta=110.683(7)^{\circ}$.

$\mathrm{c}=8.887(4) \AA$

$\gamma=90^{\circ}$.

959.1(7) $\AA^{3}$

4

$1.785 \mathrm{Mg} / \mathrm{m}^{3}$

$2.277 \mathrm{~mm}^{-1}$

532

$0.22 \times 0.08 \times 0.08 \mathrm{~mm}^{3}$

2.79 to $25.47^{\circ}$.

$-14<=\mathrm{h}<=13,0<=\mathrm{k}<=11,0<=\mathrm{l}<=10$

2938

$2738[\mathrm{R}(\mathrm{int})=0.0000]$

$98.8 \%$

Integration

0.8565 and 0.6545

Full-matrix least-squares on $\mathrm{F}^{2}$

$2738 / 3 / 78$

0.965

$\mathrm{R} 1=0.0526, \mathrm{wR} 2=0.1429$

$\mathrm{R} 1=0.0796, \mathrm{wR} 2=0.1562$

0.863 and -0.501 e. $\AA^{-3}$ 
Table S3. Atomic coordinates ( $\left.\times 10^{4}\right)$ and equivalent isotropic displacement parameters $\left(\AA^{2} \times 10^{3}\right)$ for p05yas. $U(e q)$ is defined as one third of the trace of the orthogonalized $U^{i j}$ tensor.

\begin{tabular}{lcccc}
\hline & $\mathrm{x}$ & $\mathrm{y}$ & $\mathrm{z}$ & $\mathrm{U}(\mathrm{eq})$ \\
$\mathrm{C}(1)$ & & & & \\
$\mathrm{C}(2)$ & $7400(4)$ & $4792(4)$ & $325(5)$ & $29(1)$ \\
$\mathrm{C}(3)$ & $7145(3)$ & $3719(4)$ & $1455(4)$ & $21(1)$ \\
$\mathrm{N}(1)$ & $8644(4)$ & $5324(5)$ & $939(6)$ & $41(1)$ \\
$\mathrm{O}(1)$ & $6466(3)$ & $5882(3)$ & $-62(3)$ & $20(1)$ \\
$\mathrm{O}(2)$ & $6095(2)$ & $3679(2)$ & $1474(3)$ & $22(1)$ \\
$\mathrm{O}(3)$ & $7943(2)$ & $2912(3)$ & $2260(3)$ & $28(1)$ \\
$\mathrm{Cu}(1)$ & 5000 & $6517(4)$ & 2500 & $27(1)$ \\
& 5000 & 5000 & 0 & $19(1)$ \\
\hline
\end{tabular}


Table S4. Bond lengths $[\AA]$ and angles $\left[{ }^{\circ}\right]$ for p05yas.

\begin{tabular}{|c|c|}
\hline $\mathrm{C}(1)-\mathrm{N}(1)$ & $1.483(5)$ \\
\hline $\mathrm{C}(1)-\mathrm{C}(3)$ & $1.493(7)$ \\
\hline $\mathrm{C}(1)-\mathrm{C}(2)$ & $1.540(5)$ \\
\hline $\mathrm{C}(1)-\mathrm{H}(1)$ & 1.0000 \\
\hline $\mathrm{C}(2)-\mathrm{O}(2)$ & $1.244(4)$ \\
\hline $\mathrm{C}(2)-\mathrm{O}(1)$ & $1.273(4)$ \\
\hline $\mathrm{C}(3)-\mathrm{H}(3 \mathrm{~A})$ & 0.9800 \\
\hline $\mathrm{C}(3)-\mathrm{H}(3 \mathrm{~B})$ & 0.9800 \\
\hline $\mathrm{C}(3)-\mathrm{H}(3 \mathrm{C})$ & 0.9800 \\
\hline $\mathrm{N}(1)-\mathrm{Cu}(1)$ & $1.977(3)$ \\
\hline $\mathrm{N}(1)-\mathrm{H}(1 \mathrm{~A})$ & $0.92(3)$ \\
\hline $\mathrm{N}(1)-\mathrm{H}(1 \mathrm{~B})$ & $0.899(19)$ \\
\hline $\mathrm{O}(1)-\mathrm{Cu}(1)$ & $1.958(2)$ \\
\hline $\mathrm{O}(3)-\mathrm{H}(3)$ & $0.81(2)$ \\
\hline $\mathrm{Cu}(1)-\mathrm{O}(1) \# 1$ & $1.958(2)$ \\
\hline $\mathrm{Cu}(1)-\mathrm{N}(1) \# 1$ & $1.977(3)$ \\
\hline $\mathrm{N}(1)-\mathrm{C}(1)-\mathrm{C}(3)$ & $115.4(3)$ \\
\hline $\mathrm{N}(1)-\mathrm{C}(1)-\mathrm{C}(2)$ & 108.1(3) \\
\hline$C(3)-C(1)-C(2)$ & 113.2(3) \\
\hline $\mathrm{N}(1)-\mathrm{C}(1)-\mathrm{H}(1)$ & 106.5 \\
\hline $\mathrm{C}(3)-\mathrm{C}(1)-\mathrm{H}(1)$ & 106.5 \\
\hline $\mathrm{C}(2)-\mathrm{C}(1)-\mathrm{H}(1)$ & 106.5 \\
\hline $\mathrm{O}(2)-\mathrm{C}(2)-\mathrm{O}(1)$ & $123.0(3)$ \\
\hline $\mathrm{O}(2)-\mathrm{C}(2)-\mathrm{C}(1)$ & 119.8(3) \\
\hline $\mathrm{O}(1)-\mathrm{C}(2)-\mathrm{C}(1)$ & 117.2(3) \\
\hline $\mathrm{C}(1)-\mathrm{C}(3)-\mathrm{H}(3 \mathrm{~A})$ & 109.5 \\
\hline $\mathrm{C}(1)-\mathrm{C}(3)-\mathrm{H}(3 \mathrm{~B})$ & 109.5 \\
\hline $\mathrm{H}(3 \mathrm{~A})-\mathrm{C}(3)-\mathrm{H}(3 \mathrm{~B})$ & 109.5 \\
\hline $\mathrm{C}(1)-\mathrm{C}(3)-\mathrm{H}(3 \mathrm{C})$ & 109.5 \\
\hline $\mathrm{H}(3 \mathrm{~A})-\mathrm{C}(3)-\mathrm{H}(3 \mathrm{C})$ & 109.5 \\
\hline $\mathrm{H}(3 \mathrm{~B})-\mathrm{C}(3)-\mathrm{H}(3 \mathrm{C})$ & 109.5 \\
\hline $\mathrm{C}(1)-\mathrm{N}(1)-\mathrm{Cu}(1)$ & $107.9(2)$ \\
\hline $\mathrm{C}(1)-\mathrm{N}(1)-\mathrm{H}(1 \mathrm{~A})$ & $111(2)$ \\
\hline
\end{tabular}




$\begin{array}{lc}\mathrm{Cu}(1)-\mathrm{N}(1)-\mathrm{H}(1 \mathrm{~A}) & 111(2) \\ \mathrm{C}(1)-\mathrm{N}(1)-\mathrm{H}(1 \mathrm{~B}) & 112(2) \\ \mathrm{Cu}(1)-\mathrm{N}(1)-\mathrm{H}(1 \mathrm{~B}) & 108(2) \\ \mathrm{H}(1 \mathrm{~A})-\mathrm{N}(1)-\mathrm{H}(1 \mathrm{~B}) & 108(3) \\ \mathrm{C}(2)-\mathrm{O}(1)-\mathrm{Cu}(1) & 114.3(2) \\ \mathrm{O}(1)-\mathrm{Cu}(1)-\mathrm{O}(1) \# 1 & 180.0 \\ \mathrm{O}(1)-\mathrm{Cu}(1)-\mathrm{N}(1) & 84.00(11) \\ \mathrm{O}(1) \# 1-\mathrm{Cu}(1)-\mathrm{N}(1) & 96.00(11) \\ \mathrm{O}(1)-\mathrm{Cu}(1)-\mathrm{N}(1) \# 1 & 96.00(11) \\ \mathrm{O}(1) \# 1-\mathrm{Cu}(1)-\mathrm{N}(1) \# 1 & 84.00(11) \\ \mathrm{N}(1)-\mathrm{Cu}(1)-\mathrm{N}(1) \# 1 & 180.00(15)\end{array}$

Symmetry transformations used to generate equivalent atoms:

$\# 1-x+1,-y+1,-z$ 
Table S5. Anisotropic displacement parameters $\left(\AA^{2} \times 10^{3}\right)$ for p05yas. The anisotropic displacement factor exponent takes the form: $-2 \pi^{2}\left[h^{2} a^{* 2} U^{11}+\ldots+2 h k a^{*} b^{*} U^{12}\right]$

\begin{tabular}{lcccccc}
\hline & $\mathrm{U}^{11}$ & $\mathrm{U}^{22}$ & $\mathrm{U}^{33}$ & $\mathrm{U}^{23}$ & $\mathrm{U}^{13}$ & $\mathrm{U}^{12}$ \\
\hline $\mathrm{C}(1)$ & $23(3)$ & $29(2)$ & $34(2)$ & $5(2)$ & $9(2)$ & $3(2)$ \\
$\mathrm{C}(2)$ & $20(2)$ & $21(2)$ & $18(2)$ & $-3(1)$ & $3(2)$ & $2(2)$ \\
$\mathrm{C}(3)$ & $21(3)$ & $42(3)$ & $53(3)$ & $14(2)$ & $4(2)$ & $1(2)$ \\
$\mathrm{N}(1)$ & $20(2)$ & $18(2)$ & $19(1)$ & $1(1)$ & $5(1)$ & $2(1)$ \\
$\mathrm{O}(1)$ & $18(1)$ & $26(1)$ & $23(1)$ & $6(1)$ & $6(1)$ & $2(1)$ \\
$\mathrm{O}(2)$ & $20(2)$ & $30(2)$ & $32(1)$ & $8(1)$ & $7(1)$ & $8(1)$ \\
$\mathrm{O}(3)$ & $15(2)$ & $25(2)$ & $34(2)$ & 0 & $2(2)$ & 0 \\
$\mathrm{Cu}(1)$ & $11(1)$ & $20(1)$ & $23(1)$ & $4(1)$ & $2(1)$ & $1(1)$ \\
& & & & & & \\
\hline
\end{tabular}


Table S6. Hydrogen coordinates ( $\left.\mathrm{x} 10^{4}\right)$ and isotropic displacement parameters $\left(\AA^{2} \times 10^{3}\right)$ for p05yas.

\begin{tabular}{lllll}
\hline & \multicolumn{1}{c}{$\mathrm{x}$} & $\mathrm{y}$ & $\mathrm{z}$ & $\mathrm{U}(\mathrm{eq})$ \\
& & & & \\
$\mathrm{H}(1)$ & 7294 & 4290 & -703 & 35 \\
$\mathrm{H}(3 \mathrm{~A})$ & 9193 & 4540 & 1070 & 62 \\
$\mathrm{H}(3 \mathrm{~B})$ & 8802 & 5784 & 1979 & 62 \\
$\mathrm{H}(3 \mathrm{C})$ & 8752 & 5996 & 170 & 62 \\
$\mathrm{H}(1 \mathrm{~A})$ & $6350(30)$ & $6270(40)$ & $-1060(40)$ & 29 \\
$\mathrm{H}(1 \mathrm{~B})$ & $6660(30)$ & $6580(30)$ & $670(40)$ & 29 \\
$\mathrm{H}(3)$ & $4430(30)$ & $7030(30)$ & $2310(50)$ & 40 \\
& & & & \\
\hline
\end{tabular}


Table S7. Torsion angles $\left[{ }^{\circ}\right]$ for p05yas.

\begin{tabular}{lc}
\hline $\mathrm{N}(1)-\mathrm{C}(1)-\mathrm{C}(2)-\mathrm{O}(2)$ & $-162.1(3)$ \\
$\mathrm{C}(3)-\mathrm{C}(1)-\mathrm{C}(2)-\mathrm{O}(2)$ & $-33.1(5)$ \\
$\mathrm{N}(1)-\mathrm{C}(1)-\mathrm{C}(2)-\mathrm{O}(1)$ & $20.0(4)$ \\
$\mathrm{C}(3)-\mathrm{C}(1)-\mathrm{C}(2)-\mathrm{O}(1)$ & $149.1(3)$ \\
$\mathrm{C}(3)-\mathrm{C}(1)-\mathrm{N}(1)-\mathrm{Cu}(1)$ & $-157.9(3)$ \\
$\mathrm{C}(2)-\mathrm{C}(1)-\mathrm{N}(1)-\mathrm{Cu}(1)$ & $-30.0(3)$ \\
$\mathrm{O}(2)-\mathrm{C}(2)-\mathrm{O}(1)-\mathrm{Cu}(1)$ & $-176.7(2)$ \\
$\mathrm{C}(1)-\mathrm{C}(2)-\mathrm{O}(1)-\mathrm{Cu}(1)$ & $1.1(4)$ \\
$\mathrm{C}(2)-\mathrm{O}(1)-\mathrm{Cu}(1)-\mathrm{N}(1)$ & $-15.5(2)$ \\
$\mathrm{C}(2)-\mathrm{O}(1)-\mathrm{Cu}(1)-\mathrm{N}(1) \# 1$ & $164.5(2)$ \\
$\mathrm{C}(1)-\mathrm{N}(1)-\mathrm{Cu}(1)-\mathrm{O}(1)$ & $25.5(2)$ \\
$\mathrm{C}(1)-\mathrm{N}(1)-\mathrm{Cu}(1)-\mathrm{O}(1) \# 1$ & $-154.5(2)$ \\
& \\
\hline
\end{tabular}

Symmetry transformations used to generate equivalent atoms:

$\# 1-x+1,-y+1,-z$ 
Table S8. Hydrogen bonds for p05yas [ $\AA$ and $\left.{ }^{\circ}\right]$.

\begin{tabular}{lcccc}
\hline D-H...A & d(D-H) & d(H...A & $d(D \ldots A)$ & $<($ DHA $)$ \\
\hline $\mathrm{N}(1)-\mathrm{H}(1 \mathrm{~A}) \ldots \mathrm{O}(1) \# 2$ & $0.92(3)$ & $2.10(4)$ & $2.980(4)$ & $158(3)$ \\
$\mathrm{N}(1)-\mathrm{H}(1 \mathrm{~B}) \ldots \mathrm{O}(2) \# 3$ & $0.899(19)$ & $2.15(2)$ & $3.037(4)$ & $168(3)$ \\
$\mathrm{O}(3)-\mathrm{H}(3) \ldots \mathrm{O}(2) \# 4$ & $0.81(2)$ & $1.97(3)$ & $2.758(3)$ & $164(4)$ \\
& & & & \\
\hline
\end{tabular}

Symmetry transformations used to generate equivalent atoms:

$\# 1-x+1,-y+1,-z \quad \# 2 x,-y+1, z-1 / 2 \quad \# 3-x+3 / 2, y+1 / 2,-z+1 / 2$

$\# 4 \mathrm{x}-1 / 2, \mathrm{y}+1 / 2, \mathrm{z}$ 